\title{
Dietary Intake of Medium- and Long-chain Triacylglycerols Prevents the Progression of Hyperglycemia in Diabetic ob/ob Mice
}

\author{
Shin Terada ${ }^{1 *}$, Seiji Sekine ${ }^{2}$ and Toshiaki Aoyama ${ }^{2}$ \\ ${ }^{1}$ Department of Life Sciences, Graduate School of Arts and Sciences, The University of Tokyo, 3-8-1 Komaba, Meguro-ku, Tokyo 153-8902, \\ Japan \\ ${ }^{2}$ Central Research Laboratory, The Nisshin OilliO Group, Ltd., 1 Shinmei-cho, Yokosuka, Kanagawa 239-0832, Japan
}

\begin{abstract}
We examined the effects of the dietary intake of medium- and long-chain triacylglycerol (MLCT) on hyperglycemia in diabetic ob/ob mice. Six-week-old male ob/ob mice were fed a diet containing longchain triacylglycerol (LCT) or MLCT for 3 wks. During the dietary treatment, we determined the plasma glucose and insulin concentrations in the fed state once a week. Whereas the body weights did not differ between the two groups, the total intra-abdominal fat mass was significantly higher in the MLCT group compared to the LCT group. The plasma glucose levels in the freely fed state gradually increased during the 3-wk dietary treatment in the LCT but not MLCT group, although the daily food intake did not differ between the two groups. In the fed state, the MLCT group's plasma glucose was significantly lower and their insulin concentrations were significantly higher than those observed in the LCT group ( $p<0.01)$. Plasma glucose concentrations at the end of dietary treatment (3rd wk) were negatively correlated with plasma insulin concentrations $(p<0.05)$ and tended to be inversely related to total intra-abdominal fat mass $(p=0.08)$. These results suggest that the dietary intake of MLCT may delay the progression of hyperglycemia in ob/ob mice, possibly through the stimulation of glucose uptake in intra-abdominal fat tissue caused by enhanced insulin secretion.
\end{abstract}

Key words: medium-chain fatty acids, type 2 diabetes, insulin, hyperglycemia, ob/ob mouse

\section{INTRODUCTION}

There has been an explosive increase in the prevalence of type 2 diabetes $(\mathrm{T} 2 \mathrm{D})^{1}{ }^{1}$. In light of the rapid increase in the number of elderly people around the world, T2D has become a medical and socioeconomic issue in industrialized countries. Accumulating evidence indicates that lifestyle interventions including dietary modification and exercise are associated with improved glycemic control in patients with T2D. Since eating is a major aspect of daily life, diets may have a strong influence on the development of diabetes and its subsequent progression.

It has been suggested that the quality of dietary lipids may be an important modulator of the risks associated with lifestyle-related disease. Compared with long-chain fatty acids (LCFAs), medium-chain fatty acids (MCFAs; composed of chains of $8-12$ carbon atoms) have several unique and physiologic properties. Medium-chain triacylglycerols (MCTs), composed exclusively of MCFAs, are hy- drolyzed rapidly. The resulting MCFAs are absorbed directly by the liver via the portal vein. In the liver, the MCFAs are more easily oxidized since its intramitochondrial transport does not require a carnitine transport system, which is a rate-limiting step of mitochondrial $\beta$-oxidation ${ }^{2,3)}$. These characteristics make MCTs a useful dietary treatment for preventing and reversing obesity ${ }^{3)}$. Previous studies have also reported that treatment with MCTs can improve insulin sensitivity and glucose tolerance in T2D subjects ${ }^{4,5)}$. Although MCTs may be a valuable tool for preventing and reversing obesity and T2D, it is not feasible to substitute MCTs for long-chain triacylglycerol(LCTs) in the diet for long-term therapy because the lower smoking point of MCTs and greater tendency to bubble limit the use of MCTs as cooking oil.

A cooking oil containing medium- and long-chain fatty acids in the same glycerol molecule (MLCT) prepared by random interesterification has been used widely in Japan.

*Correspondence to: Shin Terada, Department of Life Sciences, Graduate School of Arts and Sciences, The University of Tokyo, 3-8-1 Komaba, Meguro-ku, Tokyo 153-8902, Japan

E-mail: terada@idaten.c.u-tokyo.ac.jp

Accepted February 20, 2015 (received for review December 17, 2014)

Journal of Oleo Science ISSN 1345-8957 print / ISSN 1347-3352 online

http://www.jstage.jst.go.jp/browse/jos/ http://mc.manusriptcentral.com/jjocs 
MLCT has overcome the disadvantages encountered with MCT and can easily replace LCT in the daily diet. We previously reported that the dietary intake of MLCT induced a significant decrease in the mesenteric fat mass in rats fed a high-fat diet and ameliorated their whole-body insulin resistance, which precedes the onset of T2D, suggesting that MLCT can prevent the future development of T2D ${ }^{6)}$. However, it remains unclear whether MLCT is also effective for preventing and reversing the progression of hyperglycemia in patients who have already developed T2D. The purpose of the present study was thus to examine the effects of the dietary intake of MLCT on hyperglycemia in diabetic ob/ob mice, which is one of the most commonly used animal models of T2D.

\section{EXPERIMENTAL}

\subsection{Materials}

The MLCT and LCT (rapeseed oil) oils were obtained from The Nisshin OilliO Group Ltd. (Tokyo). Their fatty acid compositions, as measured by gas chromatography ${ }^{7)}$, are listed in Table 1.

\subsection{Treatment of animals}

Five-week-old male ob/ob mice were obtained from Japan SLC (Hamamatsu, Shizuoka, Japan) and were individually housed in cages. The temperature of the animal room was set at $23 \pm 1{ }^{\circ} \mathrm{C}$ with humidity of $50 \pm 5 \%$ and illumination from 09:00 to 21:00 h. All animals were treated in accordance with the guidelines for the care and use of laboratory animals (Notification of the Prime Minister's Office in Japan). The experimental plan was approved by the Laboratory Animal Care Committee of the Research Laboratory, The Nisshin OilliO Group, Ltd. During the acclimation period, the mice were allowed free access to water and

Table 1 Fatty acid compositions of the test lipids.

\begin{tabular}{|c|c|c|}
\hline Fatty acid * & LCT & MLCT \\
\hline & \multicolumn{2}{|c|}{$\mathrm{g} / 100 \mathrm{~g}$ fatty acids } \\
\hline $8: 0$ & ND & 8.4 \\
\hline $10: 0$ & ND & 2.8 \\
\hline $16: 0$ & 5.6 & 2.8 \\
\hline $18: 0$ & 2.4 & 1.2 \\
\hline $18: 1$ & 62.7 & 57.7 \\
\hline $18: 2$ & 18.5 & 18.0 \\
\hline $18: 3$ & 6.7 & 7.1 \\
\hline Others & 4.1 & 2.0 \\
\hline
\end{tabular}

LCT, long-chain triacylglycerol (rapeseed oil); MLCT, medium- and long-chain triacylglycerol; ND, not detected. *Numbers are carbon atoms : double bonds.
Table 2 Composition of the experimental diets.

\begin{tabular}{lcc}
\hline \multirow{2}{*}{\multicolumn{1}{c}{ Ingredient }} & \multicolumn{2}{c}{ Dietary group (g/kg diet) } \\
\cline { 2 - 3 } & LCT & MLCT \\
\hline Cornstarch & 367.486 & 367.486 \\
Casein & 200 & 200 \\
Alpha-cornstarch & 132 & 132 \\
Sucrose & 100 & 100 \\
LCT oil & 100 & - \\
MLCT oil & - & 100 \\
Cellulose powder & 50 & 50 \\
Mineral Mix (AIN-93G) & 35 & 35 \\
Vitamin mix (AIN-93) & 10 & 10 \\
L-Cystine & 3 & 3 \\
Choline bitartrate & 2.5 & 2.5 \\
tert-Butyl hydroquinone & 0.014 & 0.014 \\
\hline
\end{tabular}

a diet based on the AIN-93G formula.

The ob/ob mice were separated into two groups matched for body weight, plasma glucose and insulin concentrations after a 1-wk acclimation. Each group of mice was allowed free access to the experimental diet containing $\operatorname{LCT}(n=5)$ or $\operatorname{MLCT}(n=5)$ for 3 wks. The composition of the diet is presented in Table 2. Both diets were based on the AIN-93G formula with the modification that each oil was increased from $70 \mathrm{~g}$ to $100 \mathrm{~g} / \mathrm{kg}$ of the diet. Each group' s food intake and body weights were recorded every second day.

\subsection{Blood sampling}

To determine the effects of LCT intake and those of MLCT intake on plasma glucose and insulin levels in the freely fed state, we sampled the blood of each mouse between 09:00 and 09:30 in the morning (immediately after the 12-h dark period) once a week during the 3 wks of dietary treatment. The blood samples were collected into capillary tubes from the tail vein. The capillary tubes were then centrifuged and plasma samples were stored at $-80^{\circ} \mathrm{C}$ until the analysis.

\subsection{Tissue collection}

At the end of the dietary treatment (day 21), the mice were killed by bleeding under anesthesia with isoflurane. The mice were not deprived of food before sacrificing. Liver and intra-abdominal fats (epididymal, mesenteric, and retroperitoneal fat pads) were removed and weighed. Liver samples were frozen in liquid $\mathrm{N}_{2}$ and stored at $-80^{\circ} \mathrm{C}$ until the analysis.

\subsection{Analytical procedure}

Plasma glucose was determined using Glucose C2 Test 
Wako kits obtained from Wako Pure Chemical(Osaka, Japan). The plasma insulin concentration was measured by enzyme-linked immunosorbent assay (ELISA) kit according to the manufacturer's instructions (Mercodia, Uppsala, Sweden).

Liver samples were homogenized in saline and total lipids were extracted with chloroform-methanol (2:1 vol/ vol), as described by Folch et $a l^{8}$. . The lipid containing chloroform layers were separated by centrifugation and the samples were further processed using Frayn and Maycock's ${ }^{9)}$ modification of the method of Denton and Randle ${ }^{10)}$. Triacylglycerols were then quantified spectrophotometrically as glycerol using an assay kit (Triglyceride E TEST Wako). For the measurement of the glycogen concentrations, the liver was homogenized with $0.3 \mathrm{M}$ perchloric acid. The glycogen concentration was determined by enzymatic methods according to the Lowry and Passoneau method after acid hydrolysis ${ }^{11)}$.

\subsection{Statistical analysis}

The data are presented as mean \pm SEM. We performed a two-way analysis of variance (ANOVA) (Jandel Sigma Stat, San Jose, CA) to examine the effects of time and dietary conditions on plasma glucose and insulin concentrations in the fed state. We performed least-square regression analyses to examine relationships between variables. For the other experiments, the statistical analysis was performed using Student's t-test (Jandel Sigma Stat). Statistical significance was defined as $p<0.05$.

\section{RESULTS}

\subsection{Body weight, food intake, intra-abdominal fat, and liver weights}

There were no significant differences in the final body weights and body weight gains between the LCT and MLCT groups (Table 3). Food intake and food efficiency were not significantly different between the two groups (Table 3). The total intra-abdominal fat weight in the MLCT group was significantly higher than that observed in the LCT group $(p<0.05$, Table 3$)$. No significant difference in the liver weight was observed between the LCT and MLCT groups (Table 3).

\subsection{Liver triacylglycerol and glycogen concentrations}

There were no significant differences in liver triacylglycerol or glycogen concentrations between the LCT and MLCT groups $(292 \pm 32$ vs. $322 \pm 20 \mathrm{mg} / \mathrm{g}$ wet tissue, $134 \pm$ 24 vs. $113 \pm 14 \mu \mathrm{mol} / \mathrm{g}$ wet tissue, triacylglycerol and glycogen, respectively).

\subsection{Plasma glucose and insulin level in the fed state}

Figure 1 shows the changes in the plasma glucose and
Table 3 Body weight, food intake, food efficiency, and liver and intra-abdominal fat weights in the LCT and MLCT groups.

\begin{tabular}{lrc}
\hline & LCT & \multicolumn{1}{c}{ MLCT } \\
\hline Initial body weight $(\mathrm{g})$ & $34.2 \pm 1.1$ & $35.3 \pm 0.6$ \\
Final body weight $(\mathrm{g})$ & $39.4 \pm 2.1$ & $42.6 \pm 1.1$ \\
Body weight gain $(\mathrm{g})$ & $5.2 \pm 1.5$ & $7.3 \pm 0.7$ \\
Food intake $(\mathrm{g} / \mathrm{d})$ & $4.3 \pm 0.4$ & $4.0 \pm 0.2$ \\
Food efficiency $(\%)$ & $4.0 \pm 1.0$ & $5.9 \pm 0.5$ \\
Liver weight $(\mathrm{g})$ & $2.7 \pm 0.3$ & $3.3 \pm 0.1$ \\
Total intra-abdominal fat $(\mathrm{g})$ & $5.0 \pm 0.2$ & $5.6 \pm 0.1 *$ \\
\hline
\end{tabular}

The initial body weight was measured at the beginning of feeding a diet containing LCT or MLCT.

Values are presented as mean \pm SEM.

*Significant difference from the LCT group at $p<0.05$.

insulin levels in the freely fed state during the 3 wks of dietary treatment. Although the plasma glucose concentration in the fed state progressively increased in the LCT group, no such increase in plasma glucose was observed in the MLCT group (Fig. 1A). In contrast, the plasma insulin concentration progressively increased during the 3 -wk dietary treatment in the MLCT but not in the LCT group (Fig. 1B). The two-way ANOVA reveals that dietary treatment had significant effects on the plasma glucose and insulin levels in the fed state, resulting in significantly lower plasma glucose and higher plasma insulin concentrations in the MLCT group compared to the LCT group $(p<$ $0.01)$. No significant interactions between treatment duration (wk) and dietary conditions on the plasma glucose and insulin were observed.

\subsection{Relationship between plasma glucose and plasma in- sulin concentrations or total intra-abdominal fat mass}

There was a significant negative correlation between plasma insulin and glucose concentrations in the fed state at the end of dietary treatment (day 21) (Fig. 2A, r = 0.710, $p<0.05)$. As shown in Fig. $2 \mathrm{~B}$, the total intra-abdominal fat mass tended to be inversely correlated with the plasma glucose concentrations, although the relation did not reach statistical significance $(\mathrm{r}=0.575, p=0.08)$.

\section{DISCUSSION}

We previously reported that MLCT intake improves excess calorie-induced whole-body insulin resistance, which usually precedes the onset of hyperglycemia, suggesting that MLCT can prevent the development of T2D ${ }^{6)}$. For the purpose of further examining the effects of MLCT on glucose metabolism in individuals who have already de- 

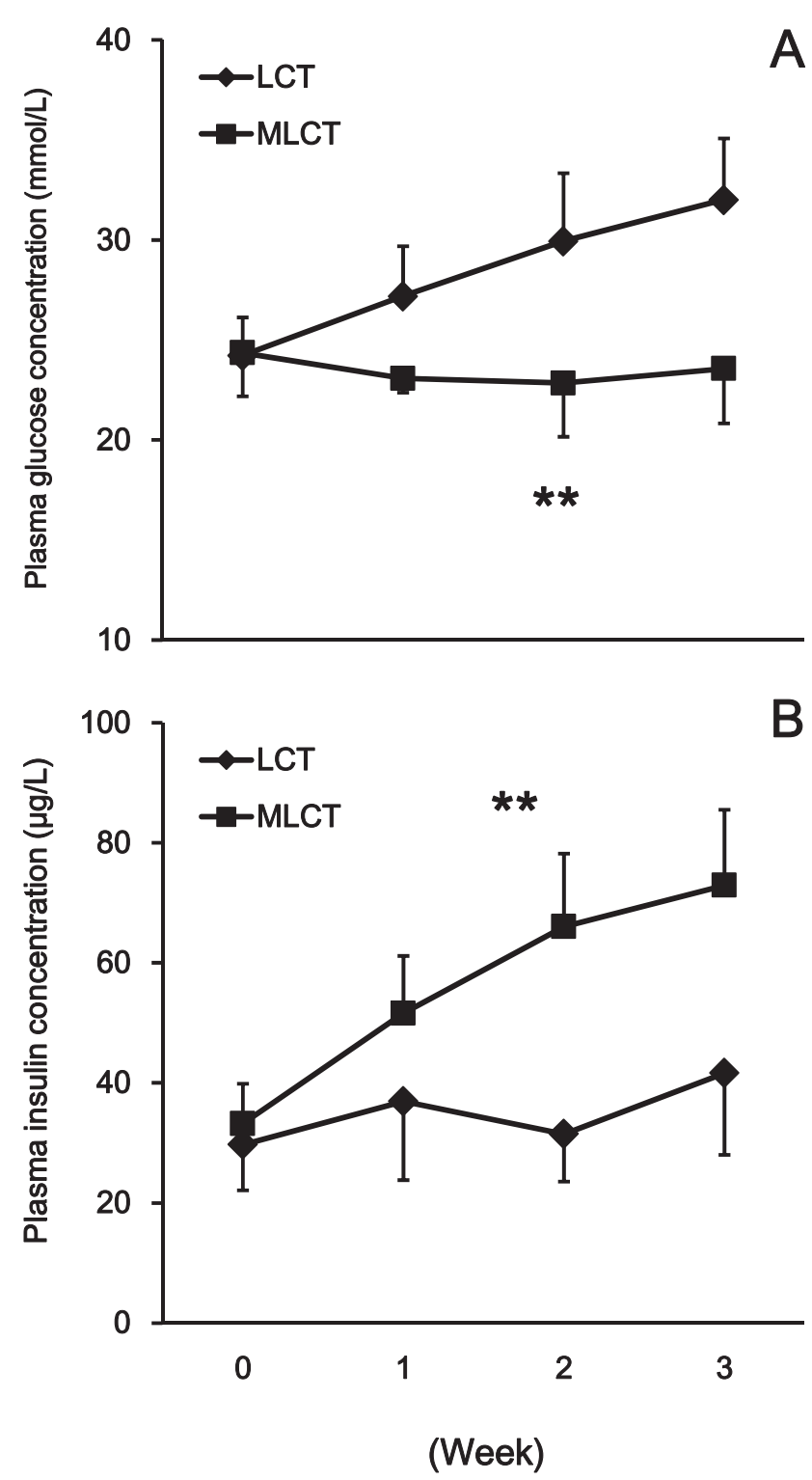

Fig. 1 Changes in plasma glucose (A) and insulin (B) concentrations in ob/ob mice fed an LCT- or MLCT-containing diet for 3 wks. Blood samples were taken in the freely fed state. Values are presented as mean \pm SEM. ${ }^{* *} p<0.01$ compared to LCT group.

veloped hyperglycemia, in the present study we evaluated the postprandial glucose levels in diabetic ob/ob mice fed a diet containing MLCT or LCT. We found that the plasma glucose level in the freely fed state gradually increased in the mice fed LCT, but not those fed MLCT (Fig. 1A), even though the daily food intake did not differ significantly between the two groups (Table 3 ). These results suggest that the dietary intake of MLCT prevents or delays the progression of hyperglycemia in T2D.

An excessive accumulation of visceral fat is postulated to
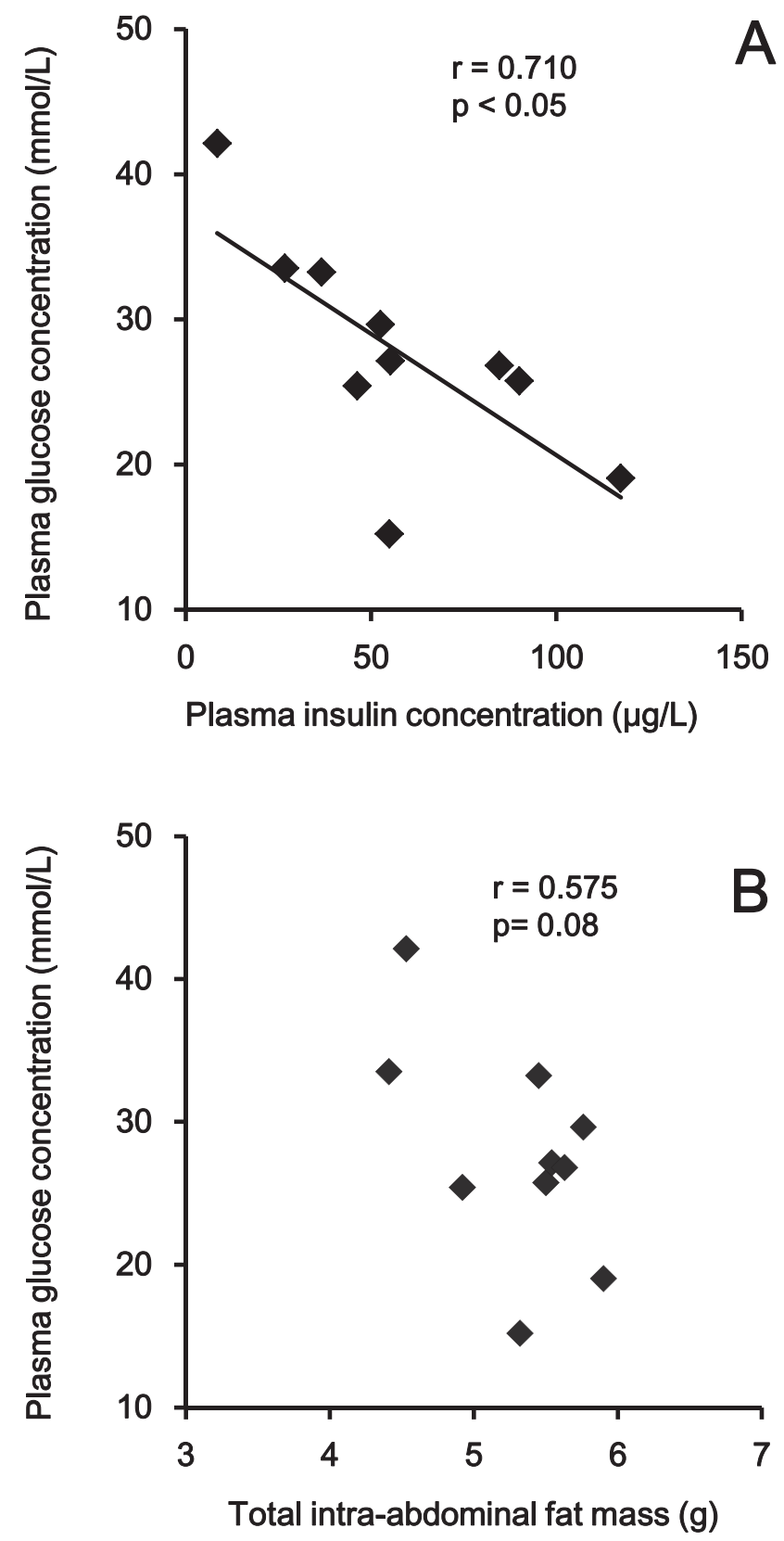

Fig. 2 Correlation between plasma glucose and insulin concentrations in the fed state at the end of the dietary treatment $(\mathrm{A})$ or total intra-abdominal fat mass in ob/ob mice fed an LCT- or MLCTcontaining diet for 3 wks.

be a major factor in the development of insulin resistance, which is an early and key defect associated with $\mathrm{T} 2 \mathrm{D}^{12)}$. It has been well documented that MLCT as well as MCT can induce a significant decrease in the visceral fat mass in both human subjects and experimental animals ${ }^{13-17)}$. We previously reported that the feeding of MLCT decreased the mesenteric fat mass in rats fed a high-fat diet, leading to the improvement of whole-body insulin resistance ${ }^{6)}$. 
Based on these findings, our working hypothesis was that the dietary intake of MLCT would induce a decrease in the total intra-abdominal fat mass in diabetic ob/ob mice as well, resulting in improved glucose homeostasis. Contrary to our expectation, the MLCT group mice in the present study had significantly higher total intra-abdominal fat masses than the LCT group (Table 3 ). In addition, the total intra-abdominal fat mass tended to be negatively associated with the plasma glucose concentration in the fed state (Fig. 2B). It is therefore plausible that under the diabetic condition, MLCT intake prevents the progression of hyperglycemia, possibly through the stimulation of glucose uptake in adipose tissue, resulting in an increase rather than a decrease in the total intra-abdominal mass, whereas MLCT may exert its body fat-lowering effects in the healthy or insulin-resistant states without hyperglycemia. However, it remains unclear whether this is a general phenomenon across species. Further studies are necessary to examine the effects of MLCT intake on the intra-abdominal fat masses in diabetic rats or human diabetic patients as well as diabetic ob/ob mice.

As shown in Fig. 1A, the plasma insulin levels in the MLCT group gradually increased during the 3 -wk dietary treatment, and a significant inverse correlation was observed between the plasma insulin and glucose concentrations (Fig. 2A). These results suggest the possibility that MLCT intake promotes insulin secretion, leading to the stimulation of glucose uptake in adipose tissue and the prevention of hyperglycemia in ob/ob mice. No such insulinotropic effects of MLCT have been reported in healthy humans or animals. It is therefore likely that MLCT exerts the insulinotropic effects only under the hyperglycemic condition.

Candidate mechanisms for the MLCT-induced increase in insulin secretion are incretin hormones such as glucagon-like peptide-1 (GLP-1) and glucose-dependent insulinotropic polypeptide (GIP). GLP-1 and GIP are hormones that are released from the gut into the bloodstream in response to the ingestion of food. They were shown to potentiate glucose-induced insulin secretion ${ }^{18)}$. Especially with respect to GIP, it has been demonstrated that orally administered fat, amino acids and glucose raised the GIP concentration in ob/ob mice, with fat having a particularly strong effect $^{19)}$. It is therefore plausible that the MLCT-induced increase in plasma insulin concentration could be attributed to an enhanced GIP secretion from the gut. Although MCT has been shown to have smaller stimulatory effects on GIP secretion compared to $\mathrm{LCT}^{20)}$, the possibility that the unique structure of MLCT (which consists of MCFAs and LCFAs in the same glycerol molecule) is effective in the stimulation of GIP release should not be ruled out. Unfortunately, we were unable to determine the plasma GIP levels in this study because the plasma samples were used up to perform the biochemical assays. Future studies are necessary to examine the effects of MLCT intake on GIP and insulin secretion.

\section{CONCLUSION}

The dietary intake of MLCT was found to have favorable effects on glycemic control in diabetic ob/ob mice. The mechanism responsible for this phenomenon appears to be, at least in part, the stimulation of glucose uptake in intra-abdominal fat tissue caused by elevated insulin secretion.

\section{References}

1) Wild, S.; Roglic, G.; Green, A.; Sicree, R.; King, H. Global prevalence of diabetes: estimates for the year 2000 and projections for 2030. Diabetes Care. 27, 1047-1053(2004).

2) Aoyama, T.; Nosaka, N.; Kasai, M. Research on the nutritional characteristics of medium-chain fatty acids. $J$. Med. Invest. 54, 385-388(2007).

3) Bach, A. C.; Babayan, V. K. Medium-chain triglycerides: an update. Am. J. Clin. Nutr. 36, 950-962 (1982).

4) Han, J. R.; Deng, B.; Sun, J.; Chen, C. G.; Corkey, B. E.; Kirkland, J. L.; Ma, J.; Guo, W. Effects of dietary medium-chain triglyceride on weight loss and insulin sensitivity in a group of moderately overweight free-living type 2 diabetic Chinese subjects. Metabolism 56, 985991 (2007).

5) Eckel, R. H.; Hanson, A. S.; Chen, A. Y.; Berman, J. N.; Yost, T. J.; Brass, E. P. Dietary substitution of mediumchain triglycerides improves insulin-mediated glucose metabolism in NIDDM subjects. Diabetes 41, 641-647 (1992).

6) Terada, S.; Yamamoto, S.; Sekine, S.; Aoyama, T. Dietary intake of medium- and long-chain triacylglycerols ameliorates insulin resistance in rats fed a high-fat diet. Nutrition 28, 92-97(2012).

7) Masood, A.; Stark, K. D.; Salem, N. Jr. A simplified and efficient method for the analysis of fatty acid methyl esters suitable for large clinical studies. J. Lipid Res. 46, 2299-2305 (2005).

8) Folch, J.; Sloane, M.; Stanley, G. H. A simple method for the isolation and purification of total lipides from animal tissues. J. Biol. Chem. 226, 497-509 (1957).

9) Frayn, K. N.; Maycock, P. F. Skeletal muscle triacylglycerol in the rat: methods for sampling and measurement, and studies of biological variability. J. Lipid Res. 21, 139-144(1980).

10) Denton, R. M.; Randle PJ. Concentrations of glycerides and phospholipids in rat heart and gastrocnemius 
muscle: effects of alloxan-diabetes and perfusion. Biochem. J. 104, 416-422 (1967).

11) Lowry, O. H.; Passoneau, J. V. A Flexible System of Enzymatic Analysis. New York: Academic, (1972).

12) Martin, B. C.; Warram, J. H.; Krolewski, A. S.; Bergman, R. N.; Soeldner, J. S.; Kahn, C. R. Role of glucose and insulin resistance in development of type 2 diabetes mellitus: results of a 25-year follow-up study. Lancet 340, 925-929 (1992).

13) Takeuchi, H.; Kubota, F.; Itakura, M.; Taguchi, N. Effect of triacylglycerols containing medium- and longchain fatty acids on body fat accumulation in rats. $J$. Nutr. Sci. Vitaminol (Tokyo). 47, 267-269 (2001).

14) Noguchi, O.; Shimada, H.; Kubota, F.; Tsuji, H., Aoyama, T. Nutritional Effects of Randomly Interesterified and Physically Mixed Oils Containing Medium-chain Fatty Acids on Rats. J. Oleo Sci. 51, 699-703 (2002).

15) Shinohara, H.; Ogawa, A.; Kasai, M.; Aoyama, T. Effect of randomly interesterified triacylglycerols containing medium- and long-chain fatty acids on energy expenditure and hepatic fatty acid metabolism in rats. Biosci. Biotechnol. Biochem. 69, 1811-1818(2005).
16) Shinohara, H.; Wu, J.; Kasai, M.; Aoyama, T. Randomly interesterified triacylglycerol containing medium- and long-chain fatty acids stimulates fatty acid metabolism in white adipose tissue of rats. Biosci. Biotechnol. Biochem. 70, 2919-2926 (2006).

17） Kasai, M.; Nosaka, N.; Maki, H.; Negishi, S.; Aoyama, T.; Nakamura, M.; Suzuki, Y.; Tsuji, H.; Uto, H.; Okazaki, M.; Kondo, K. Effect of dietary medium- and longchain triacylglycerols (MLCT) on accumulation of body fat in healthy humans. Asia Pac. J. Clin. Nutr. 12, 151-160 (2003).

18) Kim, W.; Egan, J. M. The role of incretins in glucose homeostasis and diabetes treatment. Pharmacol. Rev. 60, 470-512 (2008).

19) Flatt, P. R.; Bailey, C. J.; Kwasowski, P.; Page, T.; Marks, V. Plasma immunoreactive gastric inhibitory polypeptide in obese hyperglycaemic (ob/ob) mice. $J$. Endocrinol. 101, 249-256 (1984).

20） Kwasowski, P.; Flatt, P. R.; Bailey, C. J.; Marks, V. Effects of fatty acid chain length and saturation on gastric inhibitory polypeptide release in obese hyperglycaemic (ob/ob) mice. Biosci. Rep. 5, 701-705 (1985). 